\title{
Opposing Aminergic Modulation of Distinct Spinal Locomotor Circuits and Their Functional Coupling during Amphibian Metamorphosis
}

\author{
Aude Rauscent, James Einum, Didier Le Ray, John Simmers, and Denis Combes \\ Université de Bordeaux, Centre National de la Recherche Scientifique, Unité Mixte de Recherche 5227, 33076 Bordeaux, France
}

\begin{abstract}
The biogenic amines serotonin (5-HT) and noradrenaline (NA) are well known modulators of central pattern-generating networks responsible for vertebrate locomotion. Here we have explored monoaminergic modulation of the spinal circuits that generate two distinct modes of locomotion in the metamorphosing frog Xenopus laevis. At metamorphic climax when propulsion is achieved by undulatory larval tail movements and/or by kicking of the newly developed adult hindlimbs, the underlying motor networks remain spontaneously active in vitro, producing either separate fast axial and slow appendicular rhythms or a single combined rhythm that drives coordinated tail-based and limb-based swimming in vivo. In isolated spinal cords already expressing distinct axial and limb rhythms, bath-applied 5-HT induced coupled network activity through an opposite slowing of axial rhythmicity (by increasing motoneuron burst and cycle durations) and an acceleration of limb rhythmicity (by decreasing burst and cycle durations). In contrast, in preparations spontaneously expressing coordinated fictive locomotion, exogenous NA caused a dissociation of spinal activity into separate faster axial and slower appendicular rhythms by decreasing and increasing burst and cycle durations, respectively. Moreover, in preparations from premetamorphic and postmetamorphic animals that express exclusively axial-based or limb-based locomotion, 5-HT and NA modified the developmentally independent rhythms in a similar manner to the amines' opposing effects on the coexisting circuits at metamorphic climax. Thus, by exerting differential modulatory actions on one network that are opposite to their influences on a second adjacent circuit, these two amines are able to precisely regulate the functional relationship between different rhythmogenic networks in a developing vertebrate's spinal cord.
\end{abstract}

Key words: Xenopus laevis; locomotion; spinal networks; serotonin; noradrenaline; neuromodulation; metamorphosis; development

\section{Introduction}

The repertoires of rhythmic behaviors expressed by animals often require the functional coordination of disparate pattern-generating networks that control different muscle assemblages and operate in different temporal domains. Such coordinating interactions between distinct oscillatory networks include those responsible for walking and swimmeret movements in crustaceans (Cattaert and Clarac, 1983; Chrachri and Neil, 1993), gastric and pyloric motor rhythms in the crustacean stomatogastric system (Bartos et al., 1999; Faumont et al., 2005), and mammalian locomotion and respiration (Kawahara et al., 1989; Morin and Viala, 2002). Moreover, the extent of intercircuit interaction must be variable so that the different behaviors can be expressed independently at different frequencies, but when required, can rapidly adopt cycle frequency coupling under particular behavioral circum-

\footnotetext{
Received 0ct. 31, 2008; revised Dec. 18, 2008; accepted Dec. 23, 2008.

This work was supported by a doctoral fellowship from the Conseil Régional d'Aquitaine (A.R.). We thank Drs. Didier Morin and Romuald Nargeot for helpful discussions and comments on this manuscript.

Correspondence should be addressed to Dr. Denis Combes, Université Victor Segalen Bordeaux 2, Centre Nationa de la Recherche Scientifique, Unité Mixte de Recherche 5227, Laboratoire Mouvement Adaptation Cognition, Zone Nord, Bâtiment 2a, 146 Rue Léo Saignat, 33076 Bordeaux Cedex, France. E-mail: denis.combes@u-bordeaux2.fr. DOI:10.1523/JNEUROSCI.5255-08.2009

Copyright $\odot 2009$ Society for Neuroscience $\quad$ 0270-6474/09/291163-12\$15.00/0
}

stances (Dickinson, 1995). Although numerous studies have established the importance of neuromodulatory transmitters in conferring flexibility on individual pattern-generating networks (Marder and Calabrese, 1996; Katz, 1999; Marder and Bucher, 2007), the modulatory influences that enable distinct networks to interact dynamically to produce either coordinated or completely dissociated rhythms is significantly less well understood.

Here, we address this issue by exploring the functional relationship between rhythmogenic circuits in the spinal cord of the frog Xenopus laevis during metamorphosis when the animal undergoes a dramatic developmental transition in its mode of locomotion from the undulatory, tail-based swimming of a tadpole to limb-based kick propulsion in the young adult. At midmetamorphic stages, before tail regression, both axial and appendicular swimming can occur in the same animal, thereby indicating the coexistence of the pattern-generating networks for both locomotory strategies within the same spinal cord (Combes et al., 2004). During this transitional period, moreover, the two circuits, whether in vivo or in vitro, can produce independent rhythms with very different cycle periods or can switch spontaneously to one-to-one patterns of coordination (Rauscent et al., 2006). This flexibility in the coordination between the axial and appendicular networks therefore not only indicated that they interact function- 
ally, but also that the extent to which they interact is subject to neuromodulatory control.

The monoamines serotonin (5-HT) and noradrenaline (NA) are well documented modulators of spinal locomotory circuitry in a variety of vertebrates (Barbeau and Rossignol, 1991; Kiehn and Kjaerulff, 1996; Kiehn et al., 1999; Sqalli-Houssaini and Cazalets, 2000; Hultborn and Nielsen, 2007), including early postembryonic Xenopus larvae. In the latter, 5-HT and NA exert opposing modulatory influences on the period and duration of motoneuron bursts that drive swimming (Sillar et al., 1992, 1998) because of their differential actions on common synaptic targets within the larval axial network (McDearmid et al., 1997; McLean et al., 2000). We therefore asked whether such opposing aminergic modulation might also serve during later metamorphic development to control the motor output of coexisting axial and appendicular circuitry. By recording extracellularly from brainstem-spinal cord preparations isolated from premetamorphic, midmetamorphic, and postmetamorphic animals, we provide evidence that 5-HT and NA are able to either couple or dissociate the two distinct but behaviorally related rhythms at least partly via divergent up-modulatory or down-modulatory regulation of the underlying networks themselves.

\section{Materials and Methods}

Experiments were performed on premetamorphic, prometamorphic (at climax), and postmetamorphic stages of the South African clawed frog Xenopus laevis, bred from an in-house laboratory colony. Tadpoles were raised to appropriate stages of development [stages 50-66, according to Nieuwkoop and Faber (1956)] at room temperature. All experimental procedures were performed in conformity with the European Community Council directive of November 24, 1986 (86/609/EEC).

In vitro brainstem-spinal cord preparations. Animals were anesthetized in ice-chilled frog Ringer (composition in mM: $120 \mathrm{NaCl}, 2.5 \mathrm{KCl}, 5$ $\left.\mathrm{CaCl}_{2}, 1 \mathrm{MgCl}_{2}, \quad 15 \mathrm{NaHCO}_{3}, 7.4 \mathrm{pH}\right)$ containing $230 \mu \mathrm{g} / \mathrm{ml}$ 3 -aminobenzoic acid ethyl ester (Sigma-Aldrich) then were secured with insect pins under fresh saline in a glass Sylgard-lined Petri dish. The skin overlying the dorsal part of the brain was opened and the forebrain removed. The remaining nervous system, including the brainstem and spinal cord, was dissected free from the body, rinsed in fresh saline and then transferred to a second Petri dish for experimentation. The bath saline was equilibrated with $95 \% \mathrm{O}_{2} / 5 \% \mathrm{CO}_{2}$ and maintained at $17^{\circ} \mathrm{C}$ with a Peltier cooling system and a feedback temperature controller (HCC-100A; Dagan).

Electrophysiological recordings. Extracellular recordings of spontaneous locomotor-related activity were made with glass suction electrodes placed on selected ventral motor roots at different segmental levels along the spinal cord. Extensor and flexor motor rootlets that innervate the ankle plantaris longus (often called gastrocnemius in frogs) (Peters, 1994) and tibialis anterior, respectively, were identified by dissecting them distally to their target muscles and were recorded with stainless steel wire electrodes insulated electrically with Vaseline. Nerve signals were amplified, displayed and stored on a PC equipped with a data acquisition system (1401 CED; Cambridge Electronic Design) and analyzed off-line using Spike2 (CED) software.

Pharmacology. Serotonin (5-HT, $10 \mu \mathrm{M})$ and noradrenaline (NA, 5 $\mu \mathrm{M})$ (Sigma-RBI) were prepared from stock solutions dissolved in distilled water and used at final concentrations that were similar to those used in previous studies on postembryonic Xenopus larvae (Sillar et al., 1992; Fischer et al., 2001; Merrywest et al., 2001; McLean and Sillar, 2003, 2004). The saline containing either amine was supplied to the recording chamber at $2-5 \mathrm{ml} \cdot \mathrm{min}^{-1}$, and each application was followed by a replacement with circulating fresh saline for at least $30 \mathrm{~min}$. A Vaseline wall built across the junction between the brainstem and spinal cord allowed the amine-containing saline solution to be applied to the spinal cord alone.

Data analysis. Raw signals were first integrated and the onsets and ends of motor bursts during episodes of spontaneous locomotory-related ac- tivity in each of 10 in vitro preparations per experimental condition were automatically recognized from the recorded traces using homemade scripts running under Spike 2 software (Cambridge Electronic Design). In recordings from late prometamorphic preparations, circular statistical analyzes (Kjaerulff and Kiehn, 1996) were performed to provide a statistical measure of the degree of coupling between axial-based and limbbased activity during fictive locomotion. Individual phase values, which were obtained from a minimum of 15 cycles of stable fictive locomotion per preparation, were extracted from the latency between the onset of an axial spinal root burst and the start of the corresponding ipsilateral appendicular extensor burst and the data were transferred to Oriana software (Kovach Computing Services) for circular phase analysis. This provided a mean vector $(\mu)$ whose direction $\left(\right.$ angle $\left.{ }^{\circ}\right)$ and length $(r$, on a scale of $0-1$ ) indicated, respectively, the preferred phase and strength of coupling between bursting activity in selected axial/extensor nerve pairs. The evaluation of $r$ [using the Rayleigh test $(Z)$ to determine the concentration of phase values around the mean vector] was based on the assumption that a high concentration of phase values around the mean vector indicated strong coupling, whereas a random circular phase distribution indicated independent network activities. Coupling was considered to be significant for a probability level of $p<0.05$.

The assessment of amine-induced effects on network coupling were made using the Mardia-Watson-Wheeler test $(W$ ) to compare the preferred phase vector $\mu$ and its length $r$ before, during and after drug application for each animal ( $n=10$ per group). Pooled data provided grand means of the mean $\mu$ and $r$ for each of the 10 individual experiments of each group. Differences were again considered significant at $p<$ 0.05 .

Effects of 5-HT and NA on axial and appendicular burst parameters. The durations of individual spinal root or limb nerve bursts and their cycle periods (the latter being defined as the duration between the onsets of consecutive bursts in a given motor root or nerve) were also measured and averaged from $\geq 15$ locomotor cycles per preparation before and during application of a particular amine, and after its washout. Group values were given as means \pm SEM and differences between means were assessed by ANOVA with significance accepted at $\alpha=0.05$.

The effects of amine application on burst durations and cycle periods were compared between the axial and appendicular networks with a two-way ANOVA with the network type (appendicular vs axial) as the between-subject factor and the treatment (control, drug application and wash) as the within-subject factor. For significant amine-induced effects on the same network, a one-way ANOVA was performed separately for each network with the treatment (control, drug application and wash) remaining as the within-subject factor. In all cases, on confirmation of a significant overall effect, differences among individual means were analyzed using the Newman-Keuls post hoc test, using $p<0.05$ to accept means as statistically different.

\section{Results}

\section{Spontaneous fictive swimming in vitro}

Recordings from spinal ventral roots of in vitro brainstem-spinal cord preparations of premetamorphic, prometamorphic, and postmetamorphic Xenopus have previously shown the capacity of the isolated CNS to spontaneously generate fictive locomotor patterns at all developmental stages (Combes et al., 2004). Moreover, the in vitro motor patterns generated throughout metamorphosis are appropriate to drive the actual locomotory behavior of that particular stage. Thus, isolated preparations from premetamorphic tadpoles (e.g., the stage 52 shown in Fig. 1A) express rhythmic spinal root ( $\mathrm{sr}$ ) activity consisting of alternating bursts in the bilateral roots of each segment (see left and right sr18 recordings) that progress in a rostrocaudal sequence along the cord with a short intersegmental delay. This spontaneous in vitro activity, which occurred at a mean cycle period of $0.18 \pm 0.01 \mathrm{~s}$ in 10 measured preparations therefore corresponded to the motor pattern that would drive typical undulatory axial swimming in the intact tadpole at this stage in development (Combes et al., 2004). 


\section{A Stage 52 - premetamorphosis}
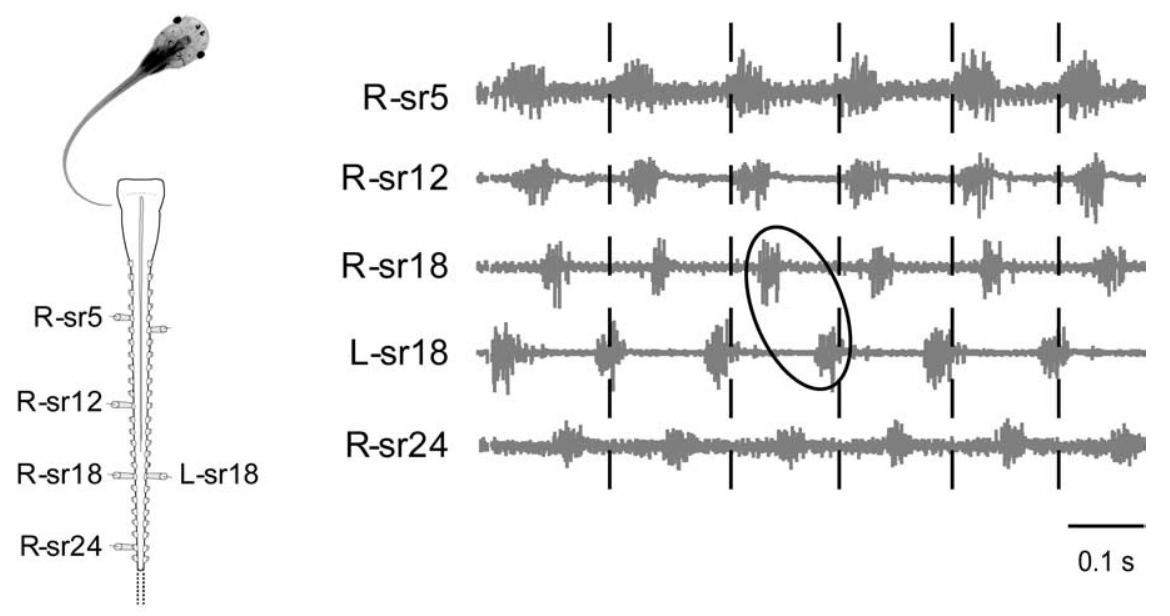

R-sr18

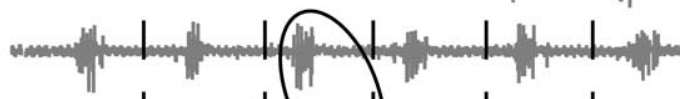

L-sr18

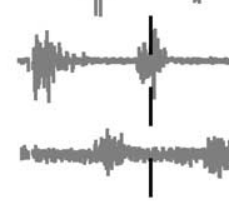

R-sr24
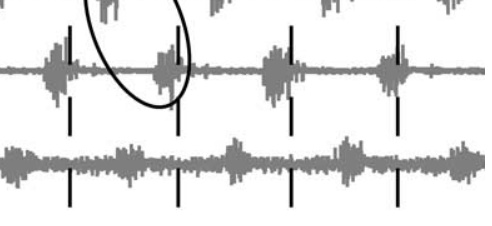

\section{B Stage 64 - postmetamorphosis}
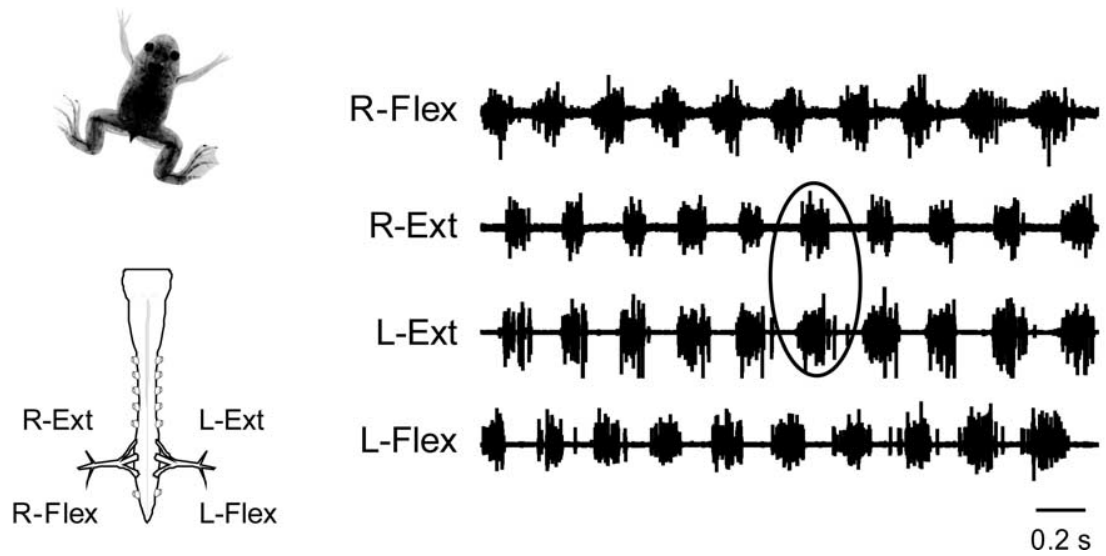

Figure 1. In vitro recordings of spontaneous fictive locomotion in isolated spinal cord/brainstem preparations of premetamorphic $(\boldsymbol{A})$ and postmetamorphic $(\boldsymbol{B})$ Xenopus laevis. $\boldsymbol{A}$, Spinal root (sr) recordings (right) at indicated segmental levels of a stage 52 tadpole (left) showing typical larval locomotor-related activity in which spontaneous axial motoneuron bursts alternate across the cord (ellipse) and progress rostrocaudally with a brief delay (vertical dotted lines indicate delay between burst onsets in right sr5 and sr24). $\boldsymbol{B}$, In a stage 64 froglet in which the tail has regressed (left) and locomotion is solely limb based, the isolated spinal cord/brainstem produces spontaneous fictive limb kicking consisting of synchronous bursts in left and right extensor (Ext) motoneurons (see ellipse) alternating with bilaterally synchronous flexor bursts.

After metamorphic climax (post-stage 65) and tail regression, propulsion is now achieved exclusively by kicking movements generated principally by the newly developed hindlimbs, and comprises short bouts of bilaterally synchronous cycles of alternating leg extensions and flexions. Correspondingly, in vitro recordings from left and right hindlimb extensor and flexor motor nerves (in our experiments those innervating the ankle plantaris longus and tibialis anterioris muscles, respectively) also displayed rhythmic burst activity (mean cycle period $0.23 \pm 0.02 \mathrm{~s}, n=10$ ) that consisted of in-phase bilateral extensor bursts alternating with synchronous bursting in left and right flexor nerves (Fig. $1 B)$. Thus the functional switch in locomotor strategy from axialbased to limb-based propulsion during metamorphosis is accompanied by a fundamental modification in the spatiotemporal organization of motor commands from left/right burst alternation along the larval spinal cord to alternating homolateral flexor-
$0.1 \mathrm{~s}$

extensor bursts that are bilaterally synchronous and originate in the lumbar cord region of the young adult (Combes et al., 2004; Rauscent et al., 2006).

During the intervening stages of metamorphosis (from stage 59 to metamorphic climax), the animal still engages in axial swimming until the tail is resorbed, although the limbs are by now fully functional and may contribute actively to body propulsion, either independently or in combination with the primary tail-based system (Combes et al., 2004). Here again, the isolated CNS of such prometamorphic animals continues to generate the rhythmic motor output patterns that correspond to the variable employment of the two different locomotory strategies in vivo. Thus, in vitro, the spinal roots and limb motor nerves may express distinctly different rhythms with the faster axial pattern sometimes occurring alone (data not shown) or, more frequently, in association with the slower appendicular rhythm (Fig. 2). In the latter situation, moreover, the two patterns may be coexpressed in independent rhythms (Fig. 2A) with completely different cycle periods, whereas at other times and even in the same preparation (albeit rarely in vitro), fictive axial and limb-based swimming can be produced in a single coordinated rhythm with bursting occurring at the same period in all recorded spinal segmental roots and hindlimb motor nerves (Fig. 2B).

This spontaneous variability in the temporal relationship between the motor output patterns for the two locomotory modes is further evident from circular statistical analysis, as typified by the two experiments in Figure 2. The random dispersion of burst onsets in R-sr12 relative to R-Ext bursts seen in the circular plot of Figure $2 A(r=0.01, Z=0.4, p>0.8 ; n=$ 10 ) and observed in $80 \%$ of the $\sim 50$ preparations used in this study was in direct contrast to the clustered phase distribution of R-sr12 versus R-Ext burst onsets $(r=0.96, Z=21.01, p<$ $0.01 ; n=10)$ during the coordinated appendicular and axial rhythmicity recorded in the remaining $20 \%$ of preparations (Fig. $2 B)$. Here, the preferential direction of the mean vector toward phase $0 / 1(\mu=0.99)$ indicated a strong tendency for burst synchronization that here, resulted from the close proximity within the spinal cord of motoneurons in the recorded sr12 and the ipsilateral lumbar extensor nerve. These in vitro observations therefore indicated that during the transitional prometamorphic period, the coexisting spinal networks that generate primary axial-based and secondary limb-based locomotory movements can spontaneously express completely dissociated (Fig. 2A) or strictly coupled (Fig. $2 B$ ) modes of coordination in correspondence with the "all-or-nothing" temporal relationship that occurs between the two swimming strategies in vivo. 


\section{Stage 61 - metamorphic climax}
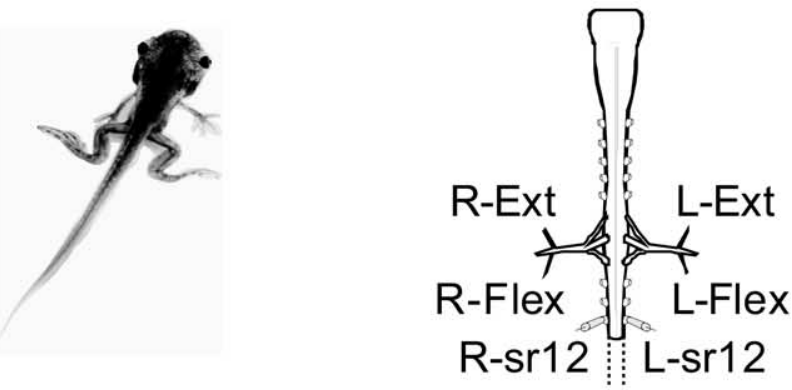

A

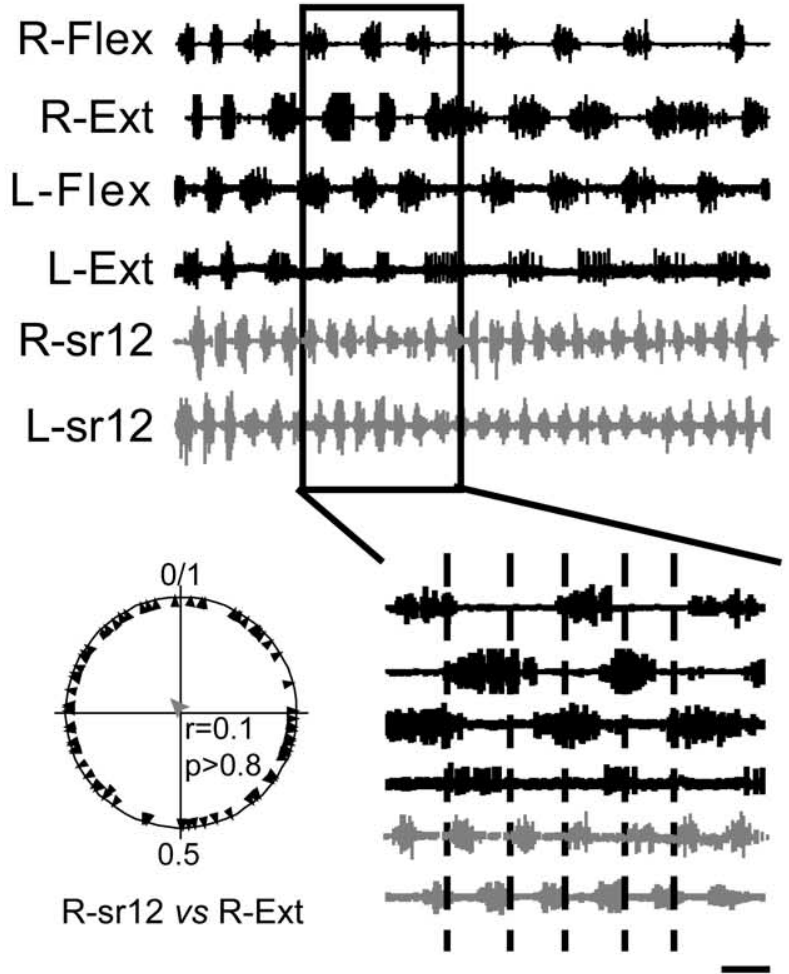

$0.1 \mathrm{~s}$

R-sr12 vs R-Ext

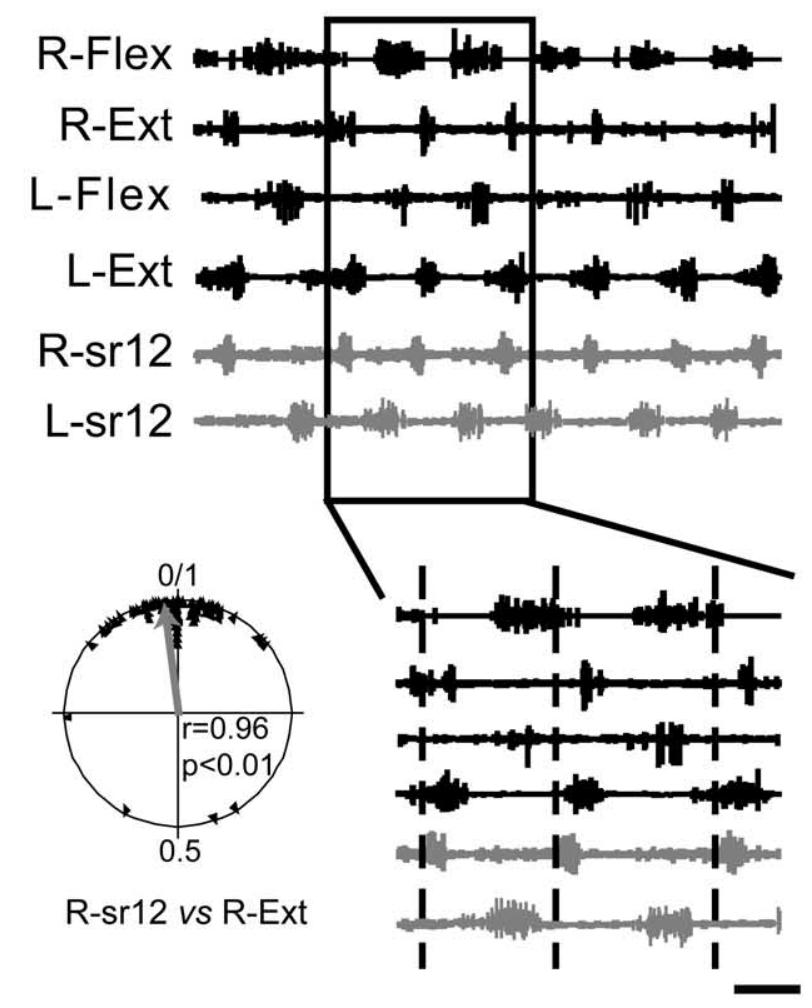

\section{R-st12 vs R-Ext}

$0.2 \mathrm{~s}$

Figure 2. Isolated CNS preparations from animals at metamorphic climax (stage 61) express both appendicular and axial fictive swimming that can be independent or coordinated in a single combined rhythm. $\boldsymbol{A}$, In $80 \%$ of in vitro preparations, spontaneous fictive limb-kicking consisting of a bilateral coactivation of flexor motor bursts in alternation with bilaterally coactive extensor bursts occur independently of axial (tail-based) network activity. In these separate rhythms, the phases of ipsilateral (right) axial sr12 burst onsets relative to right hindlimb Ext burst onsets were randomly distributed (vector length, $r=0.01$, which denotes the concentration of phase values around the mean; $p>0.8$ ), as seen in the corresponding polar plot at bottom left. $\boldsymbol{B}$, In $20 \%$ of experiments, axial and limb locomotor bursts were spontaneously generated in a single coordinated pattern, as evidenced by the tight phase coupling $(r=0.96, p<0.01)$ of burst onsets in R-sr12 versus $\mathrm{R}$-Ext in the polar plot at bottom right.

5-HT modulation of locomotor network coordination

To explore whether 5-HT is capable of modulating the coordination between these coexpressed axial and limb locomotor rhythms, we initially focused on stage 60-61 in vitro preparations $(n=10)$ that were already spontaneously producing separate axial and limb-based swimming patterns under normal saline conditions. In these cases, the independence of the two rhythms was first verified statistically by analyzing the phase relationships between recorded homolateral sr versus limb extensor burst onsets. Figure $3 A 1$ shows the control activity of one such preparation which displayed typically slower appendicular (mean period,
$1.51 \pm 0.03 \mathrm{~s}$ in this example) and faster axial $(0.21 \pm 0.01 \mathrm{~s})$ rhythms that were temporally unrelated as is evidenced by the random distribution of phase values of R-sr 15 versus R-Ext burst onsets in the corresponding circular plot $(r=0.01, Z=0.02, p>$ $0.9 ; n=30$ cycles $)$. In the presence of exogenous 5 -HT $(10 \mu \mathrm{M})$, however, substantial modifications in the ongoing fictive swimming patterns were observed (Fig. 3A2). Although the temporal structure of each rhythm remained unchanged (i.e., left-right burst alternation in the axial pattern and bilateral synchrony of hindlimb extensor motoneuron bursts), they now occurred in a single coordinated rhythm with cycle periods $(0.43 \pm 0.02)$ that 


\section{A1 Control}
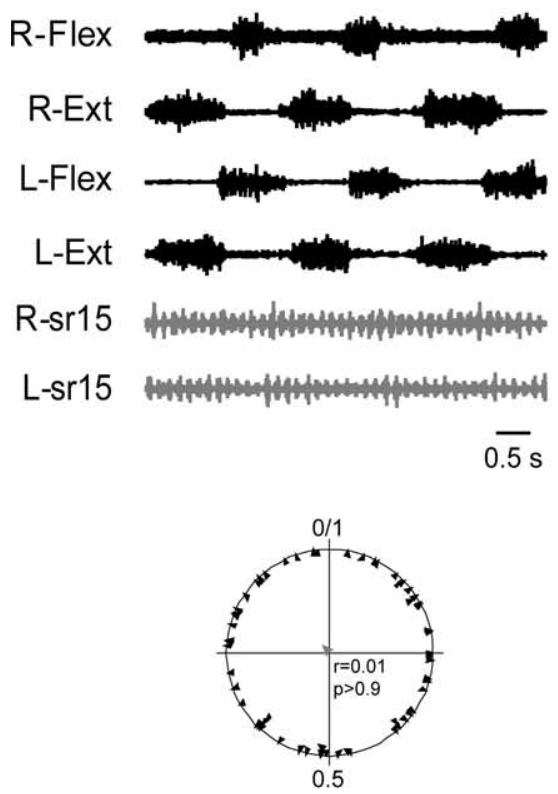

B1

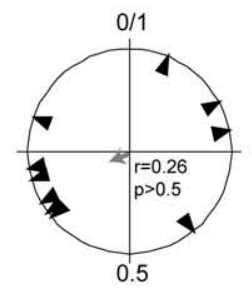

A2 $5-H T$
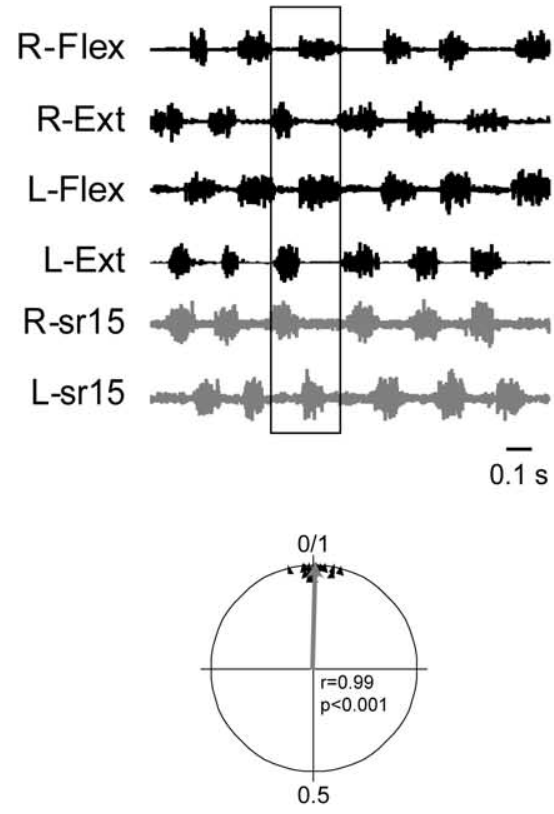

B2

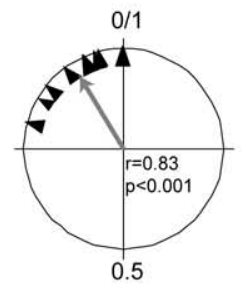

A3 Washout

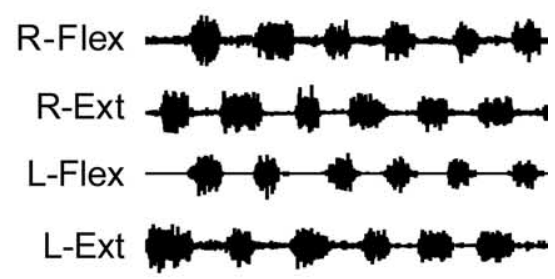

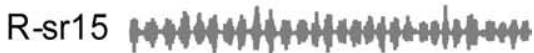

L-sr15 HHAHHHWHWHWHAHAH

$\overline{0.5 \mathrm{~s}}$

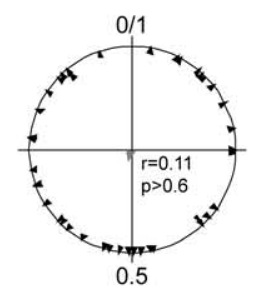

B3

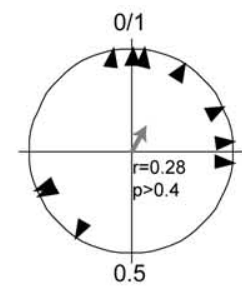

\section{R-sr15 vs R-Ext}

Figure 3. Exogenous serotonin (5-HT) couples otherwise independent axial and hindlimb locomotor rhythms into a single coordinated rhythm. $\boldsymbol{A} \boldsymbol{1}$, Initial control recordings of separate axial (sr15) and hindlimb locomotor patterns with unrelated burst-phase relationships $(r=0.01, p>0.9)$ seen in the corresponding polar plot (lower) of R-sr15 burst onsets relative to $R$-Ext burst onsets. A2, Bath application of 5 -HT $(10 \mu \mathrm{M})$ to the spinal cord of the same preparation combines the activity of the two networks into a single coupled rhythm, as indicated by the strongly significant phase preference $(r=0.99, p<0.001)$ of R-sr15 versus R-Ext burst onsets in the corresponding polar plot for this experiment. A3, The two locomotor systems return to independent activity after 5 -HT washout, as verified by the corresponding circular analysis $(r=0.11, p>0.6)$. B, Pooled data from 10 experiments (including that illustrated in $A$ ). Arrowheads denote the preferred phase direction for each experiment with the vector originating at the center point representing the grand mean of the individual mean vectors. Under 5 -HT (B2), the axial and limb locomotor rhythms were strongly coordinated $(r=0.83, p<0.001)$, in contrast to the control $(\boldsymbol{B 1} ; r=0.26, p>0.5)$ and washout $(\boldsymbol{B} 3 ; r=0.28, p>0.4)$ conditions, in which the two spinal networks operated independently.

were substantially different from those of the two original rhythms (see box in recordings of Fig. 3A2) (see below).

As is also evident from the corresponding circular plot (Fig. $3 A 2$, lower), the phase values for R-sr15 versus R-Ext were no longer uniformly distributed $(Z=14.76, p<0.001)$, but both the length $(r=0.99)$ and direction $(\mu=0.002)$ of the mean vector indicated that the axial and appendicular networks were now active in a single unified rhythm in which the analyzed sr and Ext motor bursts occurred in strict synchrony. Moreover, these effects were 5-HT-specific because the two dissociated locomotor rhythms returned after washout with normal saline (Fig. 3A3) $(r=0.11, Z=0.5, p>0.6)$. Figure $3 B$ shows pooled data from 10 similar experiments (including that of Fig. $3 A$ ), in which the arrowheads indicate the preferred discharge phases of R-sr15 versus R-Ext for individual preparations, and with the length and direction of the displayed vector $(r)$ representing the grand mean of the 10 individual mean vector lengths and directions. This group analysis clearly shows that bath-applied 5-HT reversibly
(Fig. 3B3, washout) coupled the otherwise distinct activity patterns of the two spinal locomotor networks, as indicated by the significant increase in $r$ from $0.26(Z=0.7, p>0.5)$ (Fig. 3B1) to $0.83(Z=6.9, p<0.001)$ (Fig. 3B2). The extent of coupling was statistically different $(W=17.75, p<0.001)$ between control or washout and 5-HT (pairwise analysis; $W=10.96, p<0.01$ and $W=13.376, p<0.01$, respectively), whereas no significant difference was observed between control and washout conditions $(W=0.0876, p>0.05)$.

To further understand the ability of 5-HT to coordinate spinal locomotor network activity, we examined the amine's effects on cycle period and burst duration in the two fictive swimming patterns of the 10 studied preparations. Figure 4 shows that 5-HT's presence significantly altered the cycle periods of rhythms produced by both networks $\left(F_{(2,18)}=7.84, p<0.01\right.$, for axial network bursts and $F_{(2,18)}=7.82, p<0.01$ for appendicular activity) as well as burst durations (axial: $F_{(2,18)}=11.31, p<0.001$; appendicular: $\left.F_{(2,18)}=9.87, p<0.05\right)$. Specifically, post hoc analysis 
showed that in axial spinal roots, burst cycle periods were significantly enhanced $(p<0.01)$ under 5-HT from $0.23 \pm 0.02$ to $0.33 \pm 0.003 \mathrm{~s}$ (Fig. $4 A$ ) in association with a significant $(p<0.01)$ increase in burst durations from $0.09 \pm 0.01$ to $0.13 \pm$ $0.01 \mathrm{~s}$ (Fig. $4 B$ ). In contrast, for limb extensor nerve activity, 5-HT had the opposite effect on cycle periods and burst durations (Fig. $4 A, B$ ), both of which were significantly decreased $(p<0.01$ and $p<$ 0.001 , respectively). A statistical comparison between axial and appendicular activity confirmed the differential effect of 5 -HT on the cycle periods $\left(F_{(2,36)}=9.77\right.$, $p<0.001$, two-way ANOVA) and burst durations $\left(F_{(2,36)}=11.66, p<0.001\right)$ of the two motor rhythms. Consequently, whereas these rhythm parameters of the axial and appendicular networks were significantly different in control conditions $(p<0.001$ for both cycle period and burst duration), they became statistically indistinguishable $(p>0.05$ for each parameter) under 5-HT.

These data therefore indicate that the selective application of 5-HT to the spinal cords of isolated CNS preparations coordinated their otherwise separate locomotor activity patterns into an single conjoint rhythm which was associated with a differential slowing of axial network rhythmicity, an acceleration of appendicular circuit rhythmicity, and a respective lengthening and shortening of motoneuron burst durations.

\section{NA modulation of locomotor network coordination}

Because noradrenaline has been previously shown to exert broadly opposite modulatory actions to 5-HT on axial locomotory rhythmicity in early hatchling Xenopus tadpoles (McLean et al., 2000), we assessed whether this amine might also have opposing influences on the bimodal locomotory patterns of metamorphosing animals. NA was therefore bath-applied to isolated stage 60-61 spinal cords that more rarely expressed coordinated appendicular and axial locomotor activity under control in vitro conditions, as seen previously in Figure 2 B. In 10 such preparations, the initial coupled state of locomotor network activity was first confirmed by circular analysis of the phase positions of individual R-sr15 versus R-Ext bursts, as seen in a typical single experiment in Figure 5A1 $(r>0.85, Z=55.85, p<0.001)$ and in the corresponding group analysis of Figure $5 B 1(r>0.95, Z=$ 9.03, $p<0.001)$. Again, each arrowhead in Figure $5 B$ represent the mean vector direction for one preparation, whereas the vector originating at the center point indicates the grand means of all 10 vector lengths and directions.

The wash-in of $5 \mu \mathrm{M}$ NA to the spinal cords of these preparations caused a substantial modification of their unified spontaneous rhythmicity, whereby the cycle periods of bursting in axial motoneurons decreased and periods of hindlimb motoneuron bursts increased (Fig. 5A2) (also see below) with a resultant separation of spinal activity into independent axial and appendicular rhythms. This functional dissociation of the two locomotor networks induced by NA was further evident from the now randomly dispersed phase angles of R-sr 15 versus R-Ext bursts ( $r=$ $0.06, Z=0.006, p>0.99)$ in the corresponding polar plot of the experiment shown in Figure 5A2, and in the group analysis of
B

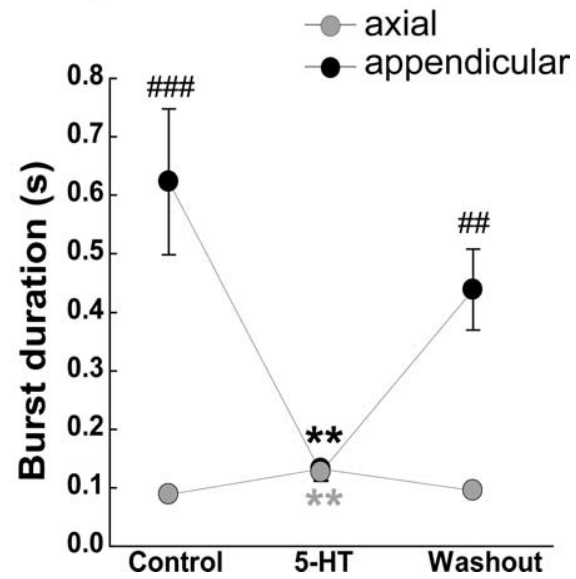

Figure 4. 5-HT modulation of axial and appendicular rhythm parameters in isolated preparations of stage 61 Xenopus. Bathapplied 5-HT significantly $\left({ }^{* *} p<0.01\right)$ and reversibly increased the overall mean cycle period $(\boldsymbol{A})$ and the mean burst duration $(\boldsymbol{B})$ ( The opposite effect was observed for conjoint hindlimb network activity (black dots) in \#\#\# ${ }^{\# \#} p<0.001$ ) or washout ( ${ }^{\# \#} p<0.01$ ), but not under 5 -HT ( $\left.p=0.95\right)$. Each point represents the grand mean of the mean cycle

Figure 5B2, in which the overall $r$ had decreased from $0.95(Z=$ 9.08, $p<0.001$ ) in initial control conditions (Fig. 5B1) to 0.19 $(Z=0.3, p>0.6)$ in the presence of NA. Moreover, the amine's circuit coordinating effects were reversible as seen in Figure $5 A 3$, $B 3$, in which as evidenced by the strongly tuned burst phase relationships in the single experiment polar plot of Figure $5 A 3(r=$ $0.85, Z=51.21, p<0.001)$ and in the group analysis of Figure $5 B 3(r=0.93, Z=8.66, p<0.001)$, coupled axial and limb locomotor activity returned after washout with normal saline. Intergroup comparisons as in Figure $5 B$ also revealed statistically significant differences $(W=19.90, p<0.001)$ between control or washout and NA $(W=8.71, p<0.05$ and $W=10, p<0.01$, respectively), whereas no difference was found between control and washout conditions ( $W=5.3, p>0.05$ ), thereby supporting the conclusion that exogenous NA was indeed responsible for causing the functional dissociation of the two spinal locomotory networks.

We next analyzed the effects of NA on the cycle periods and burst durations of axial and appendicular motor activity in the 10 analyzed preparations. Figure 6 shows that the presence of NA altered the cycle periods $\left(F_{(2,18)}=8.26, p<0.01\right.$ for axial R-sr15 and $F_{(2,18)}=26.26, p<0.001$ for hindlimb R-Ext) (Fig. 6A) and burst durations $\left(F_{(2,18)}=4.60, p<0.05\right.$ for axial R-sr15 and $F_{(2,18)}=44.04, p<0.05$ for hindlimb R-Ext) (Fig. $6 B$ ) of rhythmic activity in motoneurons belonging to the two locomotor networks. The mean burst periods of axial motoneurons decreased significantly under NA treatment from $0.32 \pm 0.04$ to $0.18 \pm 0.005 \mathrm{~s}(p<0.01)$, as did their durations from $0.09 \pm$ 0.005 to $0.07 \pm 0.004 \mathrm{~s}(p<0.05)$. In contrast, NA significantly increased both the mean cycle period and duration of limb motoneuron bursts from $0.30 \pm 0.04$ to $0.65 \pm 0.08 \mathrm{~s}(p<0.001)$ and from $0.09 \pm 0.006$ to $0.31 \pm 0.03 \mathrm{~s}(p<0.001)$, respectively. Thus, the amine exerted opposite affects on the cycle periods and burst durations of the two network rhythms $\left(F_{(2,36)}=28.75, p<\right.$ $0.001, F_{(2,36)}=46.90, p<0.001$, respectively) so that whereas each parameter was similar for axial and appendicular motoneuron bursts in the single combined rhythm expressed under con- 
A1 Control
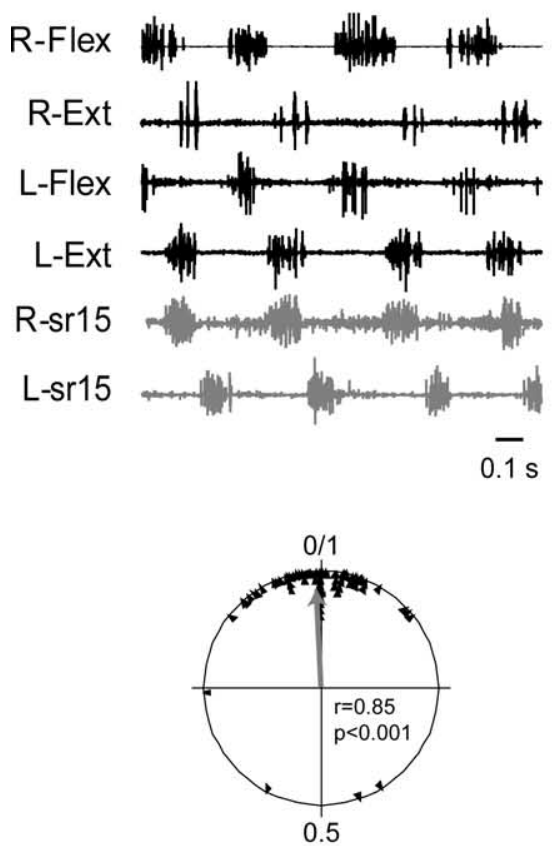

B1

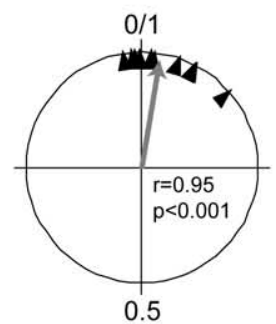

A2 NA
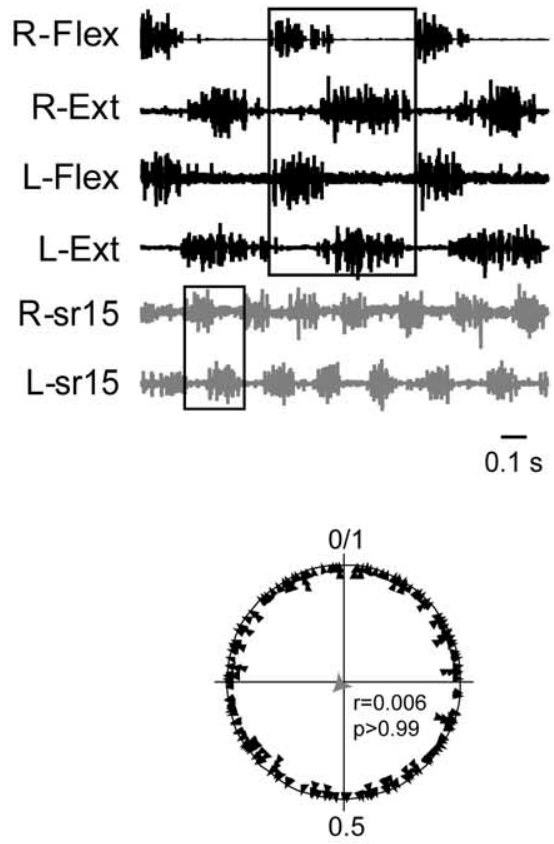

B2

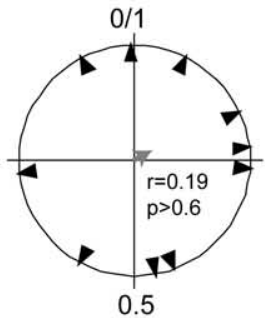

R-sr15 vs R-Ext
A3 Washout
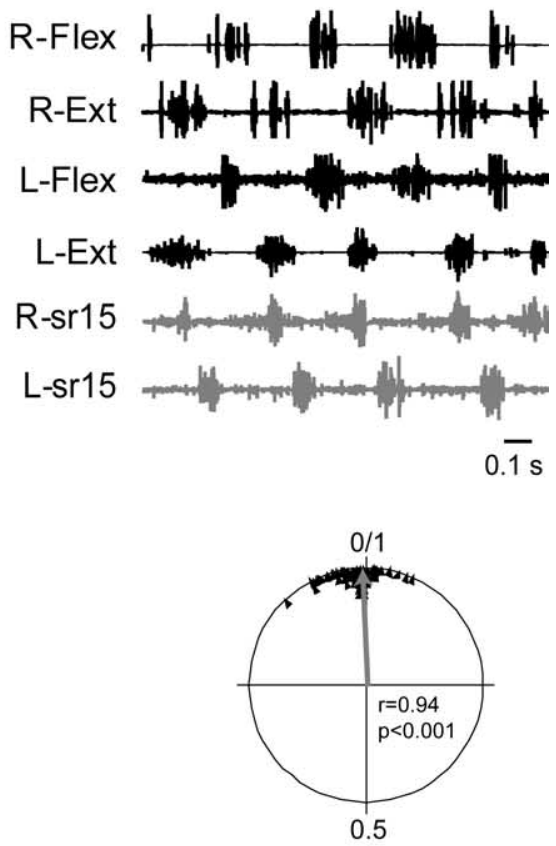

B3

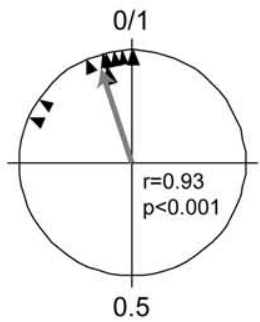

Figure 5. Exogenous noradrenaline (NA) decouples already coordinated axial and hindlimb locomotor network activity into separate rhythms. $\boldsymbol{A} 1$, In vitro recordings of axial (sr15) and hindlimb locomotor rhythms that were spontaneously coordinated, as shown by the significant phase preference $(r=0.85, p<0.001)$ of $\mathrm{R}$-sr 15 versus $\mathrm{R}$-Ext burst onsets in the corresponding polar plot below. A2, Bath application of NA ( $5 \mu \mathrm{m})$ to the spinal cord decoupled the activity of the two spinal circuits, which then operated at completely different intrinsic frequencies (see rectangles) and without any phase coupling between axial and appendicular bursts $(r=0.006, p>0.99)$, as seen in the corresponding polar plot. $A 3$, Coordinated network activity returned after the washout of $\mathrm{NA}$, as indicated by the circular plot $(r=0.94, p<0.001)$. $\boldsymbol{B}$, Pooled data from 10 experiments. Arrowheads represent the preferred phases of $\mathrm{R}$-sr 15 versus R-Ext burst onsets in $\geq 15$ cycles from each experiment, with the vector originating at the center point indicating the grand mean of the individual vectors. Under $5 \mu \mathrm{m} N \mathrm{NA}(\boldsymbol{B} 2)$, axial and hindlimb locomotor activity became segregated into two distinct rhythms $(r=0.19, p>0.6)$, unlike in the control saline $(\boldsymbol{B} 1 ; r=0.95, p<0.001)$ and washout $(\boldsymbol{B} 3 ; r=0.93, p<0.001)$ conditions, in which a single coordinated pattern was produced.

trol and washout conditions ( $p>0.05$ in both cases), they became significantly different $(p<0.001$ in each case) in the presence of NA.

These data therefore indicate that application of NA to the spinal cord dissociated the two fictive swimming rhythms through a differential acceleration of axial network bursting and a deceleration of limb circuit bursting in conjunction with a respective shortening and lengthening of axial and appendicular motoneuron burst durations.

Monoamine modulation of locomotor networks in premetamorphic and postmetamorphic tadpoles Because both amines are clearly able to differentially modulate the extent to which the axial and appendicular spinal circuits are functionally related, it is possible that 5 -HT, for example, produces coupling of the inherently faster and slower rhythms by merely strengthening the functional interactions that must exist between the two networks, with the cycle periods and burst durations of the resultant combined motor pattern representing compromises of the otherwise separate rhythms. Alternatively in this view, with already coordinated rhythms, NA could cause a weakening of the same intercircuit connections and thereby allow the re-emergence of independent axial and appendicular network activity. A further possibility is that the amines exert their influences mainly via opposing modulatory actions on the individual axial and limb circuits themselves. To assess whether intranetwork modulation does occur, we took advantage of the unique feature of Xenopus development by examining the 
amines' effects on isolated preparations from late premetamorphic and early postmetamorphic animals at a time when one locomotory strategy is expressed without the other: at stage $52-54$, locomotion remains exclusively axial-based, whereas immediately after metamorphosis ( $>$ stage $66)$, the tail has completely regressed and the appendicular system is now the solitary mechanism for animal propulsion (Fig. 1) (see also Combes et al., 2004). Figure 7 shows that 5-HT application to isolated preparations from stage 52-54 tadpoles $(n=5)$ caused a reversible increase of $\sim 150 \%$ in both cycle periods $\left(F_{(2,8)}=\right.$ 8.08, $p<0.05$ ) (Fig. 7A1,A2) and burst durations $\left(F_{(2,8)}=9.12, p<0.01\right)$ (Fig. $7 A 1, A 3)$ in the solitary motor pattern underlying tail-based swimming. The mean period and duration of spinal root bursts augmented from $0.2 \pm 0.01$ to $0.3 \pm 0.04 \mathrm{~s}$ $(p<0.05)$ and from $0.08 \pm 0.003$ to $0.13 \pm 0.02 \mathrm{~s}(p<0.05)$, respectively. In contrast, 5-HT applied to preparations of stage 66 postmetamorphic froglets $(n=5)$ caused a significant reduction compared with control or washout in both cycle periods $\left(F_{(2,8)}=8.27, p<0.05\right)$ (Fig. 7B1,B2) and burst durations $\left(F_{(2,8)}=25.27, p<0.001\right)$ (Fig. 7B1,B3) of now exclusively limb-based fictive locomotion. Here, the mean cycle period and duration of recorded extensor motor nerve bursts decreased from $0.24 \pm 0.03$ to $0.17 \pm 0.01 s(p<0.01)$ and $0.093 \pm 0.006$ to $0.05 \pm 0.006 \mathrm{~s}(p<0.001)$, respectively. Therefore, the modulatory actions of 5-HT on these developmentally distinct locomotory networks were entirely consistent with the influences observed at metamorphic climax when the circuits coexist within the same animal.

Figure 8 shows the effects of NA on the separate swimming patterns of premetamorphic (Fig. $8 A)(n=8)$ and postmetamorphic (Fig. $8 B)(n=4)$ preparations. At stage $52-54$ when functional limbs are still absent, although exogenous NA was not found to significantly affect the cycle period of axial locomotor network activity $(p=0.78)$ (Fig. 8A1,A2), it caused a significant $(p<0.001)$ and reversible decrease in axial burst durations $\left(F_{(2,14)}=123.27, p<0.001\right)$ from $0.091 \pm 0.002$ to $0.057 \pm 0.001$ $\mathrm{s}$ (Fig. $8 A 1, A 3)$. However, in stage 66 froglets, NA reversibly increased $(p<0.001)$ the mean cycle period of the now solitary appendicular rhythms from $0.24 \pm 0.03$ to $1.30 \pm 0.09 \mathrm{~s}$ (Fig. $8 B 1, B 2)\left(F_{(2,6)}=160.15, p<0.001\right)$ as well as significantly $(p<$ $0.001)$ enhancing burst durations from a mean of $0.09 \pm 0.01$ to $0.38 \pm 0.06 \mathrm{~s}$ (Fig. 8 B1,B3) $\left(F_{(2,6)}=31.36, p<0.001\right)$. Here again, therefore, the differential actions of NA on the independent axial and appendicular networks remained generally similar to the amine's effects on their coexisting equivalents in the late prometamorphic animal.

\section{Discussion}

Two previous findings motivated the experimental work reported here. First, observations and myographic recordings in freely behaving Xenopus at metamorphic climax (Beyeler et al., 2008) indicated that during this critical transitional period the animal is capable of different modes of locomotion according to how far and how fast it wishes to swim, with both the primary tail-based and secondary limb-based systems participating either independently or conjointly in swimming behavior. In the latter condition, moreover, the two locomotor modes may be expressed in distinct rhythms with very different frequencies, or when higher velocities are required, tail oscillations and rhythmic hindlimb kicking occur at the same frequency in cooperative propulsive action. Second, the motor output patterns that drive such variably coordinated behavioral rhythms continue to be spontaneously expressed by the underlying pattern-generating networks in the isolated CNS (Combes et al., 2004). Thus metamorphosing Xenopus, which lies at the interface between two of the principal locomotory strategies found in the animal kingdom, offers a unique experimental model in which the extent and functional consequences of changes in the coordination between two central pattern generators can be readily recognized both in vivo and in vitro.

Our study has shown that the separate actions of 5-HT and NA on motor output from one locomotor circuit are opposite to their influences on the other adjacent network. Moreover, each amine's effects are essentially opposite to those exerted by the other transmitter. Thus, 5-HT application to isolated preparations that are spontaneously generating independent axial-based and limb-based rhythms not only caused an acceleration of appendicular rhythmicity and a slowing of axial activity, but the amine also induced a cycle-by-cycle coupling of the two otherwise separate patterns into a single, combined rhythm. In contrast, NA had the opposite effect: during already spontaneously coupled locomotor activity, this amine's presence led to the emergence of two separate rhythms by differentially decreasing and increasing, respectively, the cycle periods of axial and limb motor burst activity. Consequently, descending serotonergic and noradrenergic pathways in the Xenopus spinal cord (Van Mier, 1986; Gonzalez et al., 1994) offer a dual "push/pull” system for controlling the temporal relationship between the two circuits during metamorphosis, conferring closely coordinated or completely independent network operation, presumably according to the behavioral demands of the animal. These amine-specific and network-specific modulatory actions on coexisting tail-based and limb-based circuitry are schematized in Figure 9A. 


\section{Stage 52-54}

A1

Control

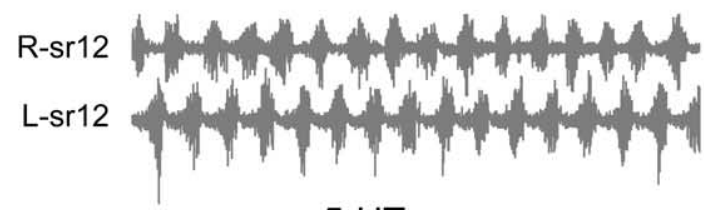

\section{5-HT}

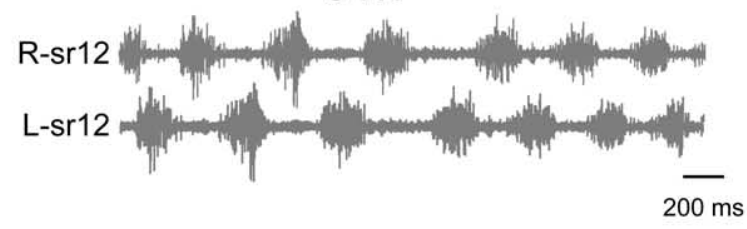

A2

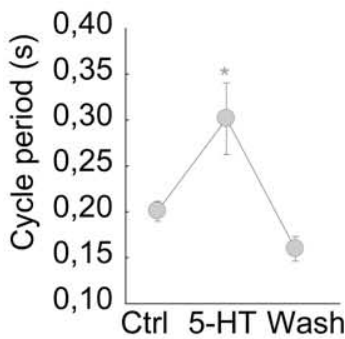

A3

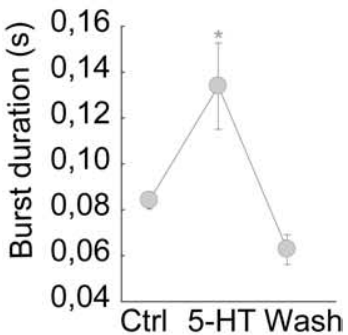

Stage 66

B1

\section{Control}

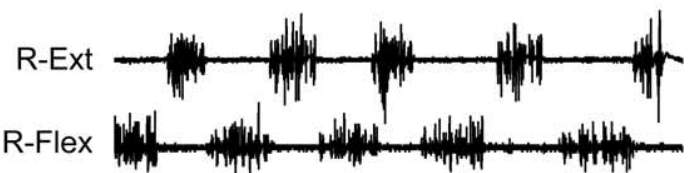

\section{5-HT}

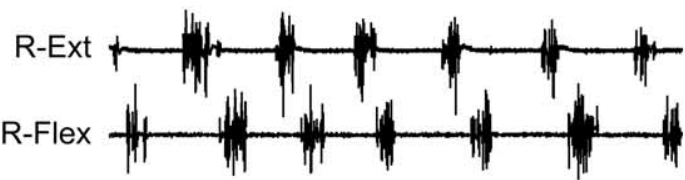

$500 \mathrm{~ms}$

\section{B2}

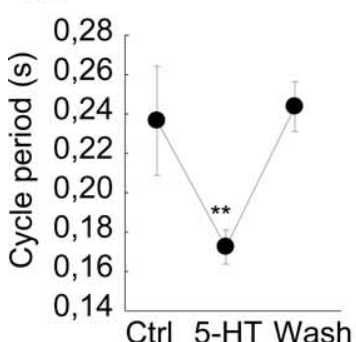

B3

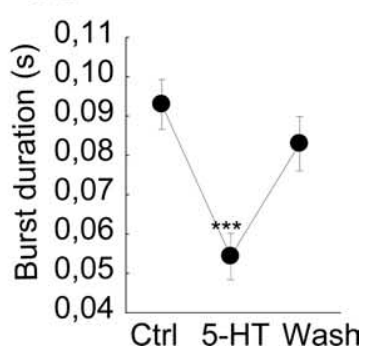

Figure 7. Opposing 5-HT modulation of axial and appendicular rhythm parameters in premetamorphic $(\boldsymbol{A})$ and postmetamorphic $(\boldsymbol{B})$ animals. $\boldsymbol{A}$, Stage $52-54$ tadpoles $(n=5)$ that exclusively use axial locomotion. $A 1$, Sample spinal root recordings from a stage 53 preparation before and after bath application of $10 \mu \mathrm{M} 5-\mathrm{HT}$. A2, A3, Group analysis of five preparations. 5 -HT significantly enhanced both the mean cycle period $\left({ }^{*} p<0.05 ; A 2\right)$ and duration $\left({ }^{*} p<\right.$ 0.05 ; $\boldsymbol{A 3})$ of sr bursts. $\boldsymbol{B}$, Stage 66 froglets $(n=5)$ that exclusively use limb-based swimming. $B 1$, Sample limb nerve recordings from a stage 66 preparation before and after 5-HT application. $\mathbf{B 2}, \mathbf{B} 3$, Group analysis $(n=5)$. 5-HT significantly decreased the mean cycle period $\left({ }^{* *} p<\right.$ $0.01 ; \boldsymbol{B} 2)$ and duration $\left({ }^{* * *} p<0.001 ; \boldsymbol{B} 3\right)$ of extensor motor nerve bursts.

\section{Stage 52-54}

A1

R-sr12

L-sr12

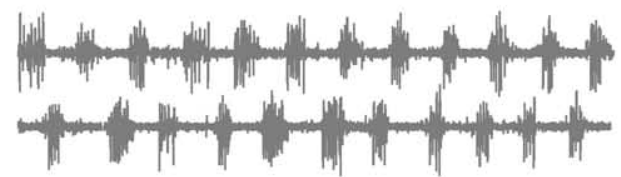

R-sr12

L-sr12

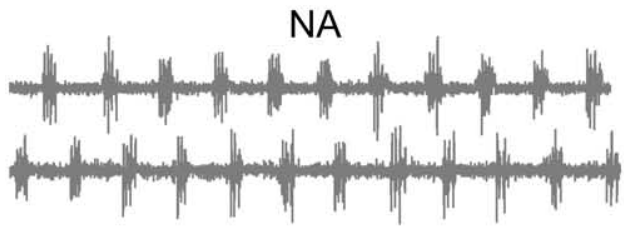

A2

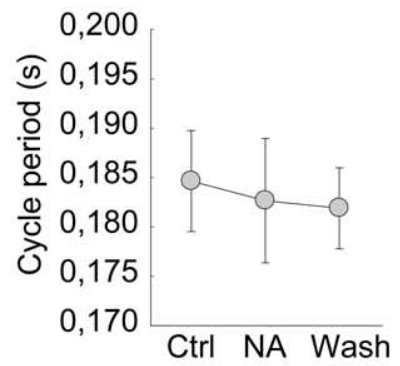

A3

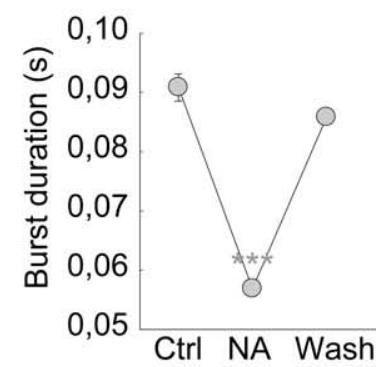

\section{Stage 66}

B1

Control

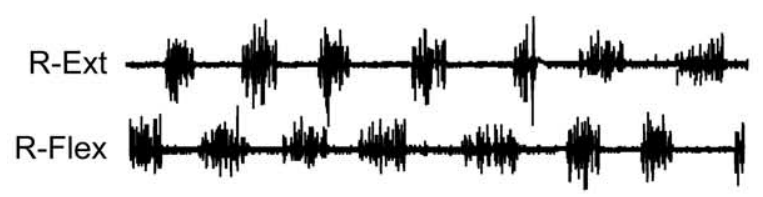

NA

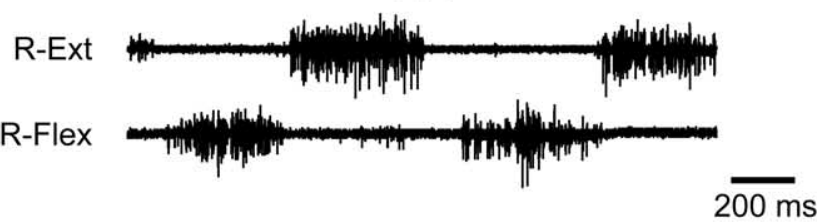

B2

B3
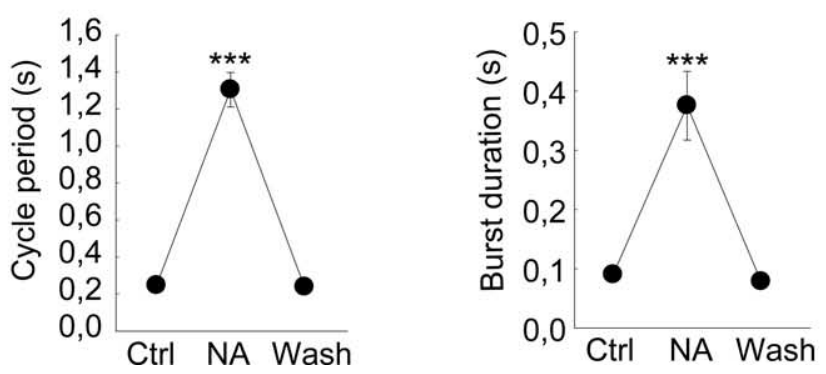

Figure 8. Opposing NA modulation of axial and appendicular rhythm parameters in premetamorphic $(\boldsymbol{A})$ and postmetamorphic $(\boldsymbol{B})$ animals. $\boldsymbol{A}$, Sample recordings from a single preparation $(\boldsymbol{A 1})$ and group analysis $(\boldsymbol{A 2}, \boldsymbol{A} \mathbf{3})$ of stage 52-54 tadpoles $(n=8)$. Bath-applied NA $(5 \mu \mathrm{m})$ did not alter the cycle period significantly $(p>0.78)$, but the mean duration of bursts was significantly and reversibly reduced $\left({ }^{* *} p<0.001\right)$. $\boldsymbol{B}$, In stage 66 froglets $(n=4$; see sample recordings in $\boldsymbol{B}$ 1), NA significantly ( ${ }^{* * *} p<0.001$ ) enhanced both the cycle periods ( $\boldsymbol{B} 2$ ) and burst durations (B3) of fictive kicking. 


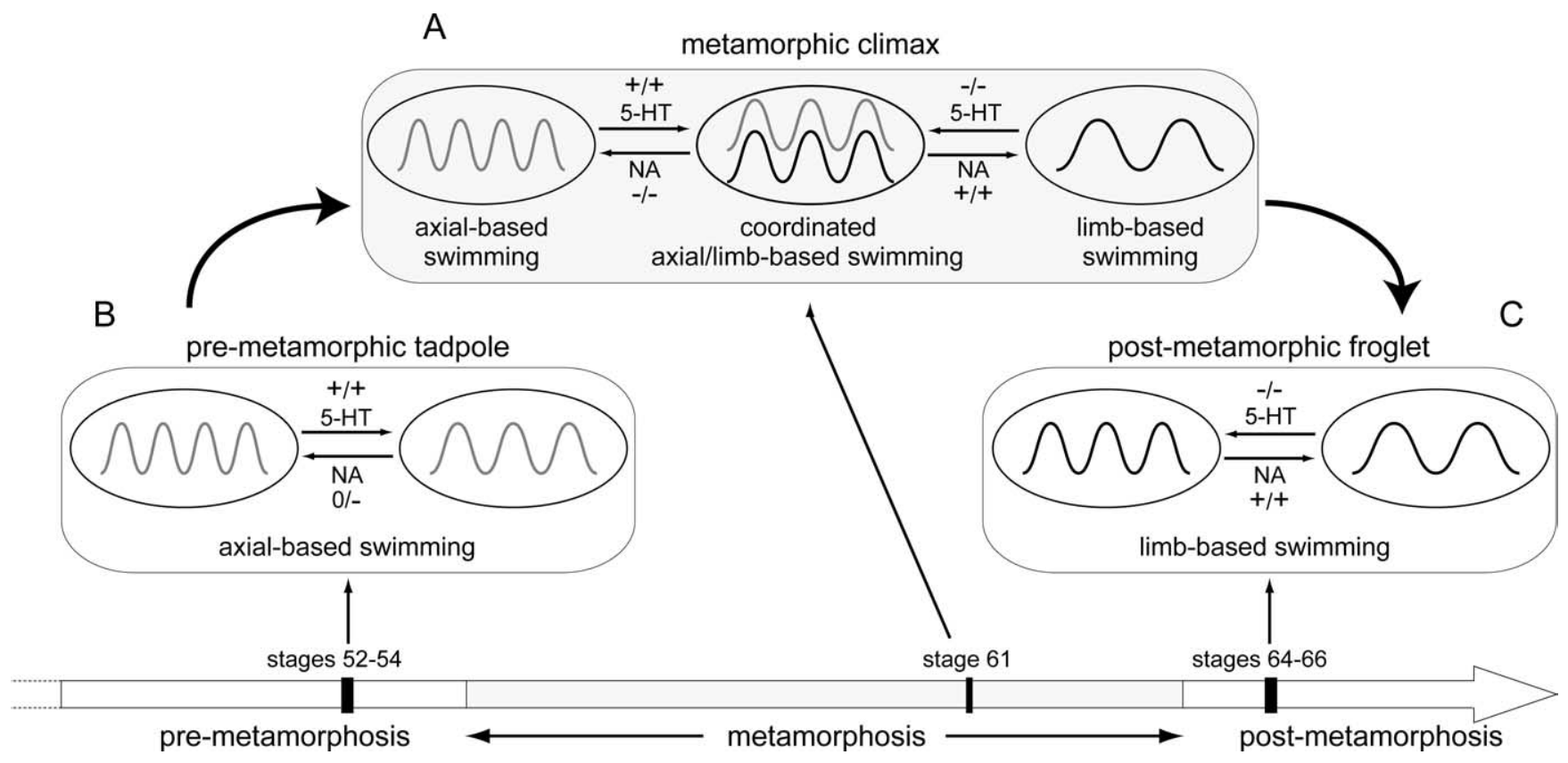

Figure 9. Summary diagrams of the differential and opposing modulatory actions of 5-HT and NA on spinal locomotor circuitry before, during, and after Xenopus metamorphosis. Symbol pairs indicate the respective effects, including an enhancement $(+)$, a reduction $(-)$, or no effect $(0)$, of the indicated monoamine on cycle period and burst duration of fictive axial-based and/or limb-based swimming. From premetamorphic stages until climax, $5-\mathrm{HT}$ increases the cycle periods and burst durations $(+/+)$ of axial locomotor network activity. NA exerts the opposite affect on the axial network at metamorphic climax $(-/-)$, although there is no evident affect on axial cycle periods $(0 /-)$ in the premetamorphic tadpole. The cycle periods and burst durations of hindlimb locomotor activity are decreased by $5-\mathrm{HT}(-/-)$ and increased by NA $(+/+)$ at climax and postmetamorphic stages. In an equivalent manner, the two amines exert opposite influences on network coupling at metamorphic climax: 5-HT combines axial and limb locomotor network activity into a single coordinated rhythm, whereas NA dissociates the two networks to allow independent rhythm generation.

Considerable information is now available on the ability of biogenic amines to modulate individual motor patterngenerating circuits (see below), although little is known about the ability of amines to regulate interacting networks with significantly different intrinsic frequencies. Opposing monoaminergic actions have also been described in motor systems including, for example, the facilitation (by 5-HT) or attenuation (by NA) of mammalian respiratory activity (Kinkead et al., 2001), and the potentiation of either recurrent inhibitory (5-HT) or excitatory (NA) spinal motor pathways (Machacek and Hochman, 2006). Moreover, a common target of differential aminergic modulation has been identified in the spinal circuitry of hatchling Xenopus embryos (McDearmid et al., 1997) in which 5-HT and NA respectively decreases or increases inhibitory glycine transmitter release from cross-cord interneurons that ensure the left-right alternation of myotomal motoneuron activity, causing opposite changes in the strength and/or frequency of axial swimming. Here again, however, these opposing aminergic influences are aimed at adapting motor performance within the context of individual networks and single behaviors.

A general principle of neuromodulation is that it provides flexibility not only via the modulation of synaptic and neuronal membrane properties within individual motor patterngenerating networks (Marder and Calabrese, 1996), but also to the synaptic connectivity between circuits (Dickinson, 1995). In metamorphosing Xenopus, the differential aminergic effects on adjacent locomotory circuitry could arise from an aminedependent increase (by 5-HT) or decrease (by NA) in the obligatory synaptic interactions that exist between the inherently faster and slower networks so that the extent of rhythm matching would be simply a function of the strength of intercircuit coupling. However, although theoretical (Nadim et al., 1998) and experimental evidence (Dickinson et al., 1990) suggests that such a mechanism could be primarily responsible for modulating network coordination, another effective way to produce the "all-ornone" network coupling seen in Xenopus would be for the modulatory process to be additionally targeted at the individual networks themselves. Indeed, that such network-directed modulatory actions occur in the Xenopus spinal cord was strongly supported by our findings that 5-HT and NA to a large extent exert the same opposing effects on the rhythm parameters of the developmentally independent fast axial and slow appendicular networks of premetamorphic or postmetamorphic animals as when the two circuits operate together during metamorphosis.

Interestingly, the one obvious difference in the aminergic effects observed in these comparative experiments also provided evidence that the interaction between the networks when they coexist is indeed also subject to modulation. Specifically, NA increased the cycle periods of axial motor bursts in midmetamorphic spinal cords (Fig. 6A) to similar values found at premetamorphosis in which NA had no effect on axial periods (Fig. 8A2). This in turn suggested that in the former under control conditions, a functional influence from the appendicular network was slowing axial bursting to produce coupled network activity, and that a weakening of this intercircuit interaction by NA allowed the axial network to escape and operate at its faster inherent frequency despite a concomitant deceleration of appendicular rhythmicity. Therefore, by taking advantage of the developmental particularity of this vertebrate model, our work points to the involvement of both intranetwork and internetwork sites of modulation in the circuit coupling/decoupling process, for which evidence has hitherto been confined to studies on the simpler nervous systems of invertebrates (Chrachri and Neil, 1993; Bartos et al., 1999; Faumont et al., 2005). 
The contrasting modulatory effects of 5-HT and NA on fictive swimming in premetamorphic tadpoles differ from their previously reported actions in the hatchling embryo. In the latter, whereas exogenously applied 5-HT increases the duration of axial motoneuron bursts with little effect on cycle periods (Sillar et al., 1992), at later premetamorphic stages, this amine increases both burst and cycle durations. In contrast, NA lengthens the cycle periods of embryonic swimming without altering motor burst durations (McDearmid et al., 1997), whereas at premetamorphosis, axial burst durations are increased but without significant changes in cycle periods (Fig. 9B). Although the functional significance of these differences is unclear, it is likely that they reflect continued maturational processes throughout the larval life of the animal (see also Brustein et al., 2003). In this sense, it is interesting that the actions of serotonin in late premetamorphic tadpoles are more closely analogous to those reported for the axial circuitry of adult undulatory swimming vertebrates such as the lamprey (Harris-Warrick and Cohen, 1985; Christenson et al., 1989) and the amphibian urodele (Branchereau et al., 2000).

In direct contrast to their respective influences on axial swimming in premetamorphic tadpoles, 5-HT decreases whereas NA increases cycle periods and burst durations of fictive appendicular locomotion in froglets (Fig. 9C). Similar serotonergic actions on locomotory circuitry occur in other limbed vertebrates. In the neonatal rat, in addition to initiating fictive locomotion in vitro (Cazalets et al., 1990, 1992), 5-HT reduces cycle periods in a concentration-dependent manner (Cazalets et al., 1990; Beato and Nistri, 1998) and enhances locomotor performance in adult rats in vivo (Feraboli-Lohnherr et al., 1999). However, in chronic spinal cats, 5-HT agonist application increases step cycle periods during treadmill walking (Barbeau and Rossignol, 1990; Rossignol et al., 1998). As we find in postmetamorphic Xenopus, NA modulates ongoing locomotor rhythmicity in the spinal cat (Rossignol et al., 1998) and the isolated neonatal rat spinal cord (Kiehn et al., 1999) by increasing both motor cycle periods and burst durations. However, it must be emphasized that our Xenopus experiments on spontaneously expressed patterns of fictive swimming differed fundamentally from most other vertebrate studies in which extant locomotor activity required sensory (e.g., via treadmill walking) or supraspinal stimulation in vivo (Steeves et al., 1980), or additional neurochemical stimulation in vitro (Grillner, 1986; Cazalets et al., 1992; Jovanovic et al., 1996; Delvolve et al., 1999; Jiang et al., 1999). Thus, distinguishing aminespecific actions from a combination of inductive/modulatory processes becomes problematic.

Finally, it is important to reiterate that our study focused primarily on a specific phase of Xenopus development, at a transitional period immediately preceding the replacement of a primary locomotory system by a completely different one. Presumably, the overlapping brainstem modulatory influences that control the cofunctional systems at metamorphic climax are also in the process of switching their downstream allegiance from axial to limb spinal circuitry before the former disappears with tail resorption. Significantly, in addition to a modulatory role, 5-HT has been implicated in the early maturation of Xenopus locomotory circuitry, and in particular, the acquisition of more efficient and flexible axial swimming in the hatchling tadpole (Sillar et al., 1993, 1995). Thus, although aminergic control of the coexisting locomotor networks described here can be seen to satisfy the immediate and rapidly changing propulsive requirements of the metamorphosing animal, it remains to be seen whether 5-HT and/or NA are additionally engaged in the longerterm development of spinal network operation as de novo limb circuitry first emerges, becomes functionally separable from, then supplants, preexisting axial swim circuitry.

\section{References}

Barbeau H, Rossignol S (1990) The effects of serotonergic drugs on the locomotor pattern and on cutaneous reflexes of the adult chronic spinal cat. Brain Res 514:55-67.

Barbeau H, Rossignol S (1991) Initiation and modulation of the locomotor pattern in the adult chronic spinal cat by noradrenergic, serotonergic and dopaminergic drugs. Brain Res 546:250-260.

Bartos M, Manor Y, Nadim F, Marder E, Nusbaum MP (1999) Coordination of fast and slow rhythmic neuronal circuits. J Neurosci 19:6650-6660

Beato M, Nistri A (1998) Serotonin-induced inhibition of locomotor rhythm of the rat isolated spinal cord is mediated by the 5-HT1 receptor class. Proc Biol Sci 265:2073-2080.

Beyeler A, Metais C, Combes D, Simmers J, Le Ray D (2008) Metamorphosis-induced changes in the coupling of spinal thoracolumbar motor outputs during swimming in Xenopus laevis. J Neurophysiol 100:1372-1383.

Branchereau P, Rodriguez JJ, Delvolve I, Abrous DN, Le Moal M, Cabelguen JM (2000) Serotonergic systems in the spinal cord of the amphibian urodele Pleurodeles waltl. J Comp Neurol 419:49-60.

Brustein E, Chong M, Holmqvist B, Drapeau P (2003) Serotonin patterns locomotor network activity in the developing zebrafish by modulating quiescent periods. J Neurobiol 57:303-322.

Cattaert D, Clarac F (1983) Influence of walking on swimmeret beating in the lobster Homarus gammarus. J Neurobiol 14:421-439.

Cazalets JR, Grillner P, Menard I, Cremieux J, Clarac F (1990) Two types of motor rhythm induced by NMDA and amines in an in vitro spinal cord preparation of neonatal rat. Neurosci Lett 111:116-121.

Cazalets JR, Sqalli-Houssaini Y, Clarac F (1992) Activation of the central pattern generators for locomotion by serotonin and excitatory amino acids in neonatal rat. J Physiol 455:187-204.

Chrachri A, Neil DM (1993) Interaction and synchronization between two abdominal motor systems in crayfish. J Neurophysiol 69:1373-1383.

Christenson J, Franck J, Grillner S (1989) Increase in endogenous 5-hydroxytryptamine levels modulates the central network underlying locomotion in the lamprey spinal cord. Neurosci Lett 100:188-192.

Combes D, Merrywest SD, Simmers J, Sillar KT (2004) Developmental segregation of spinal networks driving axial- and hindlimb-based locomotion in metamorphosing Xenopus laevis. J Physiol 559:17-24.

Delvolve I, Branchereau P, Dubuc R, Cabelguen JM (1999) Fictive rhythmic motor patterns induced by NMDA in an in vitro brain stem-spinal cord preparation from an adult urodele. J Neurophysiol 82:1074-1077.

Dickinson PS (1995) Interactions among neural networks for behavior. Curr Opin Neurobiol 5:792-798.

Dickinson PS, Mecsas C, Marder E (1990) Neuropeptide fusion of two motor-pattern generator circuits. Nature 344:155-158.

Faumont S, Combes D, Meyrand P, Simmers J (2005) Reconfiguration of multiple motor networks by short- and long-term actions of an identified modulatory neuron. Eur J Neurosci 22:2489-2502.

Feraboli-Lohnherr D, Barthe JY, Orsal D (1999) Serotonin-induced activation of the network for locomotion in adult spinal rats. J Neurosci Res 55:87-98.

Fischer H, Merrywest SD, Sillar KT (2001) Adrenoreceptor-mediated modulation of the spinal locomotor pattern during swimming in Xenopus laevis tadpoles. Eur J Neurosci 13:977-986.

Gonzalez A, Marin O, Tuinhof R, Smeets WJ (1994) Ontogeny of catecholamine systems in the central nervous system of anuran amphibians: an immunohistochemical study with antibodies against tyrosine hydroxylase and dopamine. J Comp Neurol 346:63-79.

Grillner S (1986) The effect of L-DOPA on the spinal cord related to locomotion and the half center hypothesis. In: Neurobiology and motor control (Griller S, Stein PSG, Stuart DG, Forssberg H, Herman RM, eds), pp 269-277. London: MacMillan.

Harris-Warrick RM, Cohen AH (1985) Serotonin modulates the central pattern generator for locomotion in the isolated lamprey spinal cord. J Exp Biol 116:27-46.

Hultborn H, Nielsen JB (2007) Spinal control of locomotion-from cat to man. Acta Physiol 189:111-121.

Jiang Z, Carlin KP, Brownstone RM (1999) An in vitro functionally mature 
mouse spinal cord preparation for the study of spinal motor networks. Brain Res 816:493-499.

Jovanovic K, Petrov T, Greer JJ, Stein RB (1996) Serotonergic modulation of the mudpuppy (Necturus maculatus) locomotor pattern in vitro. Exp Brain Res 111:57-67.

Katz PS (1999) Beyond neurotransmission: neuromodulation and its importance for information processing. New York: Oxford UP.

Kawahara K, Nakazono Y, Yamauchi Y, Miyamoto Y (1989) Coupling between respiratory and locomotor rhythms during fictive locomotion in decerebrate cats. Neurosci Lett 103:326-330.

Kiehn O, Kjaerulff O (1996) Spatiotemporal characteristics of 5-HT and dopamine-induced rhythmic hindlimb activity in the in vitro neonatal rat. J Neurophysiol 75:1472-1482.

Kiehn O, Sillar KT, Kjaerulff O, McDearmid JR (1999) Effects of noradrenaline on locomotor rhythm-generating networks in the isolated neonatal rat spinal cord. J Neurophysiol 82:741-746.

Kinkead R, Bach KB, Johnson SM, Hodgeman BA, Mitchell GS (2001) Plasticity in respiratory motor control: intermittent hypoxia and hypercapnia activate opposing serotonergic and noradrenergic modulatory systems. Comp Biochem Physiol A Mol Integr Physiol 130:207-218.

Kjaerulff O, Kiehn O (1996) Distribution of networks generating and coordinating locomotor activity in the neonatal rat spinal cord in vitro: a lesion study. J Neurosci 16:5777-5794.

Machacek DW, Hochman S (2006) Noradrenaline unmasks novel selfreinforcing motor circuits within the mammalian spinal cord. J Neurosci 26:5920-5928.

Marder E, Bucher D (2007) Understanding circuit dynamics using the stomatogastric nervous system of lobsters and crabs. Annu Rev Physiol 69:291-316.

Marder E, Calabrese RL (1996) Principles of rhythmic motor pattern generation. Physiol Rev 76:687-717.

McDearmid JR, Scrymgeour-Wedderburn JF, Sillar KT (1997) Aminergic modulation of glycine release in a spinal network controlling swimming in Xenopus laevis. J Physiol 503:111-117.

McLean DL, Sillar KT (2003) Spinal and supraspinal functions of noradrenaline in the frog embryo: consequences for motor behaviour. J Physiol 551:575-587.

McLean DL, Sillar KT (2004) Divergent actions of serotonin receptor activation during fictive swimming in frog embryos. J Comp Physiol A Neuroethol Sens Neural Behav Physiol 190:391-402.

McLean DL, Merrywest SD, Sillar KT (2000) The development of neuro- modulatory systems and the maturation of motor patterns in amphibian tadpoles. Brain Res Bull 53:595-603.

Merrywest SD, Fischer H, Sillar KT (2001) Alpha-adrenoreceptor activation modulates swimming via glycinergic and GABAergic inhibitory pathways in Xenopus laevis tadpoles. Eur J Neurosci 15:375-383.

Morin D, Viala D (2002) Coordinations of locomotor and respiratory rhythms in vitro are critically dependent on hindlimb sensory inputs. J Neurosci 22:4756-4765.

Nadim F, Manor Y, Nusbaum MP, Marder E (1998) Frequency regulation of a slow rhythm by a fast periodic input. J Neurosci 18:5053-5067.

Nieuwkoop P, Faber B (1956) Normal tables for Xenopus laevis. Amsterdam: North Holland Publishing Company.

Peters SE (1994) Properties of twitch motor units of the ankle extensor muscles in the bullfrog Rana catesbeiana. J Morphol 221:121-131.

Rauscent A, Le Ray D, Cabirol-Pol MJ, Sillar KT, Simmers J, Combes D (2006) Development and neuromodulation of spinal locomotor networks in the metamorphosing frog. J Physiol Paris 100:317-327.

Rossignol S, Chau C, Brustein E, Giroux N, Bouyer L, Barbeau H, Reader TA (1998) Pharmacological activation and modulation of the central pattern generator for locomotion in the cat. Ann N Y Acad Sci 860:346-359.

Sillar KT, Wedderburn JFS, Simmers AJ (1992) Modulation of swimming rhythmicity by 5 -hydroxytriptamine during post-embryonic development in Xenopus laevis. Proc Biol Sci 250:107-114.

Sillar KT, Wedderburn JFS, Woolston AM, Simmers AJ (1993) Control of locomotor movements during vertebrate development. News Physiol Sci 8:107-111.

Sillar KT, Woolston AM, Wedderburn JF (1995) Involvement of brainstem serotonergic interneurons in the development of a vertebrate spinal locomotor circuit. Proc Biol Sci 259:65-70.

Sillar KT, Reith CA, McDearmid JR (1998) Development and aminergic neuromodulation of a spinal locomotor network controlling swimming in Xenopus larvae. Ann N Y Acad Sci 860:318-332.

Sqalli-Houssaini Y, Cazalets JR (2000) Noradrenergic control of locomotor networks in the in vitro spinal cord of the neonatal rat. Brain Res 852:100-109.

Steeves JD, Schmidt BJ, Skovgaard BJ, Jordan LM (1980) Effect of noradrenaline and 5-hydroxytryptamine depletion on locomotion in the cat. Brain Res 185:349-362.

Van Mier P (1986) The development of the motor system in the clawed toad Xenopus laevis. $\mathrm{PhD}$ thesis, University of Nijmengen. 